\title{
Влияние курсового приема L-орнитин- L-аспартата на фиброз и стеатоз печени у больных ассоциированной с нарушениями метаболизма жировой болезнью печени (неалкогольной жировой болезнью печени),
} имеющих гипераммониемию

\author{
Е.В. Гаранина \\ ООО «Европейский медицинский центр «УГМК - Здоровье», г. Екатеринбург, Российская Федерация
}

\begin{abstract}
Введение. Гипераммониемия выявляется не только при циррозе печени, но также на более ранних стадиях фиброза при ассоциированной с нарушениями метаболизма жировой болезни печени (МАЖБП). Аммиак помимо нейротропного имеет также гепатотоксический и профибротический эффект. L-орнитин-L-acпартат (LOLA) показал свою эффективность в лечении гипераммониемии у больных с циррозом печени.

Цель исследования. Оценить влияние курсового приема LOLA на биомаркеры воспаления, стеатоза и фи-
\end{abstract} броза печени при МАЖБП.

Материалы и методы. 90 пациентов были разделены на 2 группы: контрольную (стеатоз печени S0-S1, отсутствие фиброза печени, нормальные показатели печеночных проб, отсутствие заболеваний печени в анамнезе) и группу пациентов с МАЖБП (стеатоз печени S2-S3) и фиброзом F1 по шкале METAVIR. CTеатоз и фиброз определяли на аппарате «Фиброскан 502» с функцией САР. Всем пациентам проведено определение аммиака в цельной крови. При повышении содержания аммиака в крови назначали препарат LOLA в дозировке 9 г в сутки на протяжении 8 недель с контрольным измерением в крови уровней аммиака, АСТ, АЛТ, ГГТП, СРБ и ферритина, а также выраженности фиброза и стеатоза после курса терапии.

Результаты. В исследовании участвовали 45 пациентов с МАЖБП и 45 в контрольной группе. Гипераммониемия была выявлена у 26 (58 \%) больных из группы МАЖБП и у 3 (7 \%) в группе контроля $(p<0,001)$. Среди пациентов с МАЖБП и гипераммониемией чаще был выявлен сахарный диабет 2-го типа и более выраженный стеатоз печени, была значимо выше доля мужчин, чем при МАЖБП без гипераммониемии. После 8 недель терапии препаратом LOLA уровни АЛТ, АСТ, ГГТП, ферритина и СРБ значимо снизились, содержание аммиака в крови вошло в нормальный диапазон ( $p<0,001)$. У 22 пациентов (85 \%) произошло уменьшение жесткости печени по данным эластометрии, причем у 6 из них эти значения стали соответствовать F0 ( $p<0,001)$. Степень стеатоза уменьшилась у 18 (69 \%) участников.

Заключение. LOLA нормализует содержание аммиака в крови, уменьшает выраженность воспаления, стеатоза и фиброза печени.

Ключевые слова: неалкогольная жировая болезнь печени, аммиак крови, стеатоз, фиброз, фиброскан с функцией САР

Конфликт интересов: Исследование выполнено при поддержке фармацевтической компании «Мерц».

Для цитирования: Гаранина E.B. Влияние курсовогоприемa L-орнитин-L-аспартатана фиброз истеатоз печени убольных ассоциированной с нарушениями метаболизма жировой болезнью печени (неалкогольной жировой болезнью печени), имеющих гипераммониемию. Российский журнал гастроэнтерологии, гепатологии, колопроктологии. 2021;31(4):31-36. https://doi.org/10.22416/1382-4376-2021-31-4-31-36

\section{Effect of L-Ornithine-L-Aspartate on Liver Fibrosis and Steatosis in Patients with Metabolic-Associated Fatty Liver Disease (Non-alcoholic Fatty Liver Disease) and Hyperammonaemia}

Elena V. Garanina

"UGMK - Zdorovye" European Medical Centre LLC, Ekaterinburg, Russian Federation 
Introduction. Hyperammonaemia develops both in cirrhosis and earlier fibrotic stages during metabolic-associated fatty liver disease (MAFLD). Besides neurotropic, ammonia exerts the hepatotoxic and profibrotic effects. L-ornithine-L-aspartate (LOLA) has been proved effective in treatment for hyperammonaemia in cirrhosis patients.

Aim. An impact study of the LOLA course therapy on inflammation, steatosis and liver fibrosis biomarkers in MAFLD. Materials and methods. A total of 90 patients were divided between two cohorts. The control cohort included patients with liver steatosis S0-S1, absent liver fibrosis, normal liver function tests, clean history of liver disease, while MAFLD cohort gathered liver steatosis S2-S3 and METAVIR fibrosis F1. Steatosis and fibrosis were assessed with a Fibroscan 502 unit with CAP measurement. All patients had ammonia estimated from whole blood. At high ammonia, LOLA was ordered at $9 \mathrm{~g} /$ day for 8 weeks, with control of blood ammonia, AST, ALT, GGT, CRP and ferritin, as well as fibrosis and steatosis post-therapy.

Results. The study enrolled 45 patients of the MAFLD and 45 - of control cohort. Hyperammonaemia was revealed in $26(58 \%)$ MAFLD and $3(7 \%)$ control patients $(p<0.001)$. MAFLD-hyperammonaemic patients also had the significantly higher male ratio, type 2 diabetes and severer hepatic steatosis rates vs. hyperammonaemia-negative MAFLD individuals. In 8 weeks of LOLA therapy, the ALT, AST, GGT, ferritin and CRP levels decreased significantly, and blood ammonia attained normal range $(p<0.001)$. Elastometry liver stiffness decreased in $22(85 \%)$ patients, reaching F0 values in 6 cases $(p<0.001)$. The steatosis grade reduced in $18(69 \%)$ individuals.

Conclusion. LOLA normalises blood ammonia levels and reduces the severity of inflammation, steatosis and liver fibrosis.

Keywords: non-alcoholic fatty liver disease, blood ammonia, steatosis, fibrosis, CAP elastometry

Conflict of interest: the work was carried out with support from Merz Pharmaceuticals.

For citation: Garanina E.V. Effect of L-Ornithine-L-Aspartate on Liver Fibrosis and Steatosis in Patients with Metabolic-Associated Fatty Liver Disease (Non-alcoholic Fatty Liver Disease) and Hyperammonaemia. Russian Journal of Gastroenterology, Hepatology, Coloproctology. 2021;31(4):31-36. https://doi.org/10.22416/1382-4376-2021-31-4-31-36

\section{Введение}

Длительное время считалось, что гипераммониемия при хронических болезнях печени имеет место только на стадии цирроза, а сам аммиак рассматривался только как нейротоксин. Однако было показано, что повышение содержания этого вещества в крови наблюдается еще до наступления цирроза, а само оно оказывает, помимо нейротропного, прямой гепатотоксичный эффект и профибротическое действие, а также подавляет функцию нейтрофилов и играет важную роль в патогенезе саркопении [1-6]. Так, при жировой болезни печени, ассоциированной с метаболическими нарушениями (МАЖБП), которая ранее определялась как неалкогольная жировая болезнь печени (НАЖБП), аммиак еще на стадии стеатоза индуцирует образование активных форм кислорода, снижает клеточный метаболизм, уменьшает активность эндотелиальной NO-синтазы (eNOS), усиливает пролиферацию звездчатых клеток и способствует таким образом активации процессов фиброза и нарушению внутрипеченочной гемодинамики [1-6]. Кроме того, гипераммониемия негативно влияет на центральную и периферическую нервную системы, вызывает гепатогенную слабость даже на ранних стадиях МАЖБП, а также может приводить к снижению работоспособности скелетных мышц с появлением «периферической» слабости [1-6].

Для коррекции гипераммониемии при заболеваниях печени длительное время применяется L-орнитина-L-аспартат (LOLA), который активирует работу орнитинового цикла детоксикации. Применение LOLA у пациентов с МАЖБП снижает выраженность цитолиза и дислипидемии $[7,8]$. Однако еще не было изучено, может ли применение этого препарата уменьшить выраженность стеатоза и фиброза печени.

Цель настоящего исследования - оценить влияние курсового приема LOLA на биомаркеры воспаления, стеатоза и фиброза печени при МАЖБП.

\section{Материалы и методы}

В исследование были включены пациенты, обратившиеся в клинику «УГМК - Здоровье» и подписавшие информированное согласие на участие в нем. Наличие других заболеваний печени (например, вирусный или аутоиммунный гепатит) и злоупотребление алкоголем служили критерием исключения из исследования. Включенные пациенты были поделены на 2 группы: контрольную (стеатоз печени S0-S1, отсутствие фиброза печени, нормальные показатели печеночных проб, отсутствие заболеваний печени в анамнезе) и группу пациентов с МАЖБП (стеатоз печени S2-S3) и фиброзом F1 по шкале METAVIR.

Стеатоз и фиброз определяли на аппарате «Фиброскан 502» с функцией САР датчиками М и XL. Результат оценивали по шкале валидизации для МАЖБП, где уровень фиброза F1 - это плотность печени с 6,1 до 7,5 кРа и уровни стеатоза S2 и S3 - это значения 269-300 и и выше 300дцБ соответственно.

Всем пациентам проведено определение аммиака в цельной крови методом микродиффузии с помощью портативного анализатора PocketChem BA PA 41-40 (ARKRay, Япония). Принцип его действия состоит в следующем. Ионы аммония из образца крови (20 мкл) при нанесении на индикаторную полоску, пропитанную солью борной кислоты, переходят в газообразное состояние 
и, попадая на индикатор (бромкрезоловый зеленый), изменяют его цвет. Степень изменения цвета пропорциональна концентрации образовавшегося аммиака (диапазон измерения - 7-286 мкмоль/л, время выполнения теста - 180 с). У ровень аммиака определяли у всех пациентов при включении в исследование и после завершения курса терапии LOLA, у пациентов с его первичным повышением. В качестве верхней границы нормального уровня аммиака крови было принято значение 60 мкмоль/л, повышение уровня аммиака 61100 мкмоль/л соответствовало критериям легкой гипераммониемии, 101-200 мкмоль/л - гипераммониемии средней степени, более 200 мкмоль/л выраженной гипераммониемии.

Всем пациентам с МАЖБП были даны рекомендации по модификации образа жизни, питания и физическим нагрузкам. При повышении содержания аммиака в крови назначали препарат LOLA в дозировке 9 г в сутки на протяжении 8 недель с контрольным измерением в крови уровней аммиака, АСТ, АЛТ, ГГТП, СРБ и ферритина, а также выраженности фиброза и стеатоза после курса терапии.

Данные представлены в виде среднего \pm среднее квадратическое отклонение. Статистическую значимость оценивали с помощью критериев Стьюдента для непрерывных переменных и хиквадрат (с поправкой Йейтса при количестве наблюдений в группе менее 6) для категориальных переменных. Значение $p<0,05$ было критерием значимости.

\section{Результаты исследования}

В исследование были включены 90 пациентов. Не было значимой разницы между группами в возрасте участников и уровне холестерина крови. Среди больных МАЖБП доля женщин была значимо больше, чем в группе контроля. При МАЖБП чаще выявлены ожирение и сахарный диабет 2-го типа, было выше значение индекса массы тела (ИМТ) и содержание гликированного гемоглобина в крови (табл. 1).

Таблица 1. Основные характеристики включенных пациентов

\begin{tabular}{|l|c|c|c|}
\hline \multicolumn{1}{|c|}{ Показатель } & Контрольная группа $(n=45)$ & МАЖБП $(n=45)$ & $p$ \\
\hline Возраст, лет & $46 \pm 10$ & $45 \pm 10$ & 0,636 \\
\hline Мужчины $/$ Женщины, $n$ & $33 / 12$ & $16 / 29$ & $<0,001$ \\
\hline ИМТ, кг $/ \mathrm{m}^{2}$ & $25,2 \pm 4,7$ & $32,2 \pm 4,7$ & $<0,001$ \\
\hline Ожирение, $n(\%)$ & 0 & $30(67 \%)$ & $<0,001$ \\
\hline Абдоминальное ожирение, $n(\%)$ & $20(44 \%)$ & $45(100 \%)$ & $<0,001$ \\
\hline Сд 2-го типа, $n$ (\%) & 0 & $7(16 \%)$ & 0,018 \\
\hline Гликированный гемоглобин, \% & $5,1 \pm 1,3$ & $5,8 \pm 1,3$ & 0,012 \\
\hline Холестерин, ммоль $/$ л & $5,4 \pm 1,8$ & $5,7 \pm 2,1$ & 0,469 \\
\hline Стеатоз печени $\mathrm{S} 0, n(\%)$ & $41(91 \%)$ & 0 & - \\
\hline Стеатоз печени $\mathrm{S} 1, n(\%)$ & $4(9 \%)$ & 0 & - \\
\hline Стеатоз печени S2, $n(\%)$ & 0 & $31(69 \%)$ & - \\
\hline Стеатоз печени S3, $n(\%)$ & 0 & $14(31 \%)$ & - \\
\hline
\end{tabular}

Table 1. Main patient profile

\begin{tabular}{|l|c|c|c|}
\hline \multicolumn{1}{|c|}{ Criteria } & Control cohort $(n=45)$ & MAFLD $(n=45)$ & $p$ \\
\hline Age, years & $46 \pm 10$ & $45 \pm 10$ & 0.636 \\
\hline Males $/$ Females, $n$ & $33 / 12$ & $16 / 29$ & $<0.001$ \\
\hline BMI, $\mathrm{kg} / \mathrm{m}^{2}$ & $25.2 \pm 4.7$ & $32.2 \pm 4.7$ & $<0.001$ \\
\hline Obesity, $n(\%)$ & 0 & $30(67 \%)$ & $<0.001$ \\
\hline Abdominal obesity, $n(\%)$ & $20(44 \%)$ & $45(100 \%)$ & $<0.001$ \\
\hline Type 2 diabetes, $n(\%)$ & 0 & $7(16 \%)$ & 0.018 \\
\hline Glycated haemoglobin, \% & $5.1 \pm 1.3$ & $5.8 \pm 1.3$ & 0.012 \\
\hline Cholesterol, mmol/L & $5.4 \pm 1.8$ & $5.7 \pm 2.1$ & 0.469 \\
\hline Liver steatosis S0, $n(\%)$ & $41(91 \%)$ & 0 & - \\
\hline Liver steatosis S1, $n(\%)$ & $4(9 \%)$ & 0 & - \\
\hline Liver steatosis S2, $n(\%)$ & 0 & $31(69 \%)$ & - \\
\hline Liver steatosis S3, $n(\%)$ & 0 & $14(31 \%)$ & - \\
\hline
\end{tabular}


Гипераммониемия была выявлена у 26 пациентов $(57,8 \%$; ДИ $=37,7-84,6 \%)$ с МАЖБП: у 20 из них она была легкой степени, а у $6-$ средней (с максимальным содержанием аммиака в крови 153 мкмоль/л). В контрольной группе повышение уровня аммиака в крови было обнаружено только у 3 человек $(6,7 \%$, ДИ $=1,4-19,5 \%$; $p<0,001)$.

Среди пациентов с МАЖБП и гипераммониемией чаще выявляли сахарный диабет 2-го типа и был более выраженный стеатоз печени, чем при МАЖБП без гипераммониемии. Несмотря на то что доли пациентов с ожирением значимо не различались между этими группами больных МАЖБП, ИМТ был выше при наличии гипераммониемии. Также у пациентов с гипераммониемией была выше активность в крови АЛТ, АСТ, ГГТ и уровень ферритина (табл. 2).

После 8 недель терапии препаратом LOLA уровни АЛТ, АСТ, ГГТП, ферритина и СРБ значимо снизились. У 22 пациентов (85 \%) произошло уменьшение плотности печени по данным «Фиброскана», причем у 6 из них эти значения стали соответствовать F0 (нет фиброза) $(p<0,001)$. Степень стеатоза уменьшилась у 18 (69 \%) человек (табл. 3).

Таблица 2. Характеристика пациентов с МАЖБП в зависимости от наличия гипераммониемии

\begin{tabular}{|l|c|c|c|}
\hline \multicolumn{1}{|c|}{ Показатель } & $\begin{array}{c}\text { Гипераммониемия } \\
\text { присутствует }(n=26)\end{array}$ & $\begin{array}{c}\text { Гипераммониемия отсутствует } \\
(n=19)\end{array}$ & $p$ \\
\hline Возраст, лет, среднее \pm СО & $49 \pm 10$ & $48 \pm 10$ & 0,742 \\
\hline Мужчины / Женщины, $n(\%)$ & $14 / 12$ & $2 / 17$ & 0,007 \\
\hline ИМТ, кг $/ \mathrm{M}^{2}$, среднее \pm СО & $33,2 \pm 4,7$ & $30,0 \pm 2,9$ & 0,007 \\
\hline Ожирение, $n(\%)$ & $16(61 \%)$ & $14(74 \%)$ & 0,594 \\
\hline СРБ, мг $/$ л & $4,1 \pm 2,1$ & $3,0 \pm 1,1$ & 0,028 \\
\hline СД 2-го типа, $n$ (\%) & $7(27 \%)$ & 0 & $<0,001$ \\
\hline Гликированный гемоглобин, \% & $6,1 \pm 1,9$ & $5,7 \pm 1,1$ & 0,379 \\
\hline Холестерин, ммоль/л & $6,2 \pm 2,1$ & $5,8 \pm 0,9$ & 0,390 \\
\hline Стеатоз печени S2, $n(\%)$ & $14(54 \%)$ & $17(89 \%)$ & 0,026 \\
\hline Стеатоз печени S3, $n(\%)$ & $12(46 \%)$ & $2(11 \%)$ & $<0,001$ \\
\hline АСТ, МЕ $/$ л & $45,0 \pm 6,7$ & $35,0 \pm 5,6$ & $<0,001$ \\
\hline АЛТ, МЕ $/$ л & $61,0 \pm 3,5$ & $52,0 \pm 7,2$ & $<0,001$ \\
\hline ГГТП, МЕ $/$ л & $89,0 \pm 7,8$ & $61,0 \pm 3,2$ & $<0,001$ \\
\hline Ферритин, мкг $/$ л & $127,0 \pm 3,1$ & $110,0 \pm 5,2$ & \\
\hline
\end{tabular}

Table 2. MAFLD patient profile by presence of hyperammonaemia

\begin{tabular}{|l|c|c|c|}
\hline \multicolumn{1}{|c|}{ Criteria } & $\begin{array}{c}\text { Hyperammonaemia } \\
(n=26)\end{array}$ & $\begin{array}{c}\text { No hyperammonaemia } \\
(n=19)\end{array}$ & $p$ \\
\hline Age, years, mean \pm SD & $49 \pm 10$ & $48 \pm 10$ & 0.742 \\
\hline Males/Females, $n(\%)$ & $14 / 12$ & $2 / 17$ & 0.007 \\
\hline BMI, kg/m², mean \pm SD & $33.2 \pm 4.7$ & $30.0 \pm 2.9$ & 0.007 \\
\hline Obesity, $n(\%)$ & $16(61 \%)$ & $14(74 \%)$ & 0.594 \\
\hline CRP, mg/L & $4.1 \pm 2.1$ & $3.0 \pm 1.1$ & 0.028 \\
\hline Type 2 diabetes, $n(\%)$ & $7(27 \%)$ & 0 & $<0.001$ \\
\hline Glycated haemoglobin, \% & $6.1 \pm 1.9$ & $5.7 \pm 1.1$ & 0.379 \\
\hline Cholesterol, mmol/L & $6.2 \pm 2.1$ & $5.8 \pm 0.9$ & 0.390 \\
\hline Liver steatosis S2, $n(\%)$ & $14(54 \%)$ & $17(89 \%)$ & 0.026 \\
\hline Liver steatosis S3, $n(\%)$ & $12(46 \%)$ & $2(11 \%)$ & $<0.001$ \\
\hline AST, IU/L & $45 \pm 6.7$ & $35 \pm 5.6$ & $<0.001$ \\
\hline ALT, IU/L & $61 \pm 3.5$ & $52 \pm 7.2$ & $<0.001$ \\
\hline GGT, IU/L & $89 \pm 7.8$ & $61 \pm 3.2$ & $<0.001$ \\
\hline Ferritin, ug/L & $127 \pm 3.1$ & $110 \pm 5.2$ & \\
\hline
\end{tabular}


Таблища 3. Динамика основных биомаркеров у пациентов с МАЖБП, имеющих гипераммониемию, после применения L-орнитина-L-аспартата

\begin{tabular}{|c|c|c|c|}
\hline Показатель & До терапии & После терапия & $p$ \\
\hline Аммиак в крови, мкмоль/л & $93,0 \pm 2,1$ & $53,0 \pm 4,1$ & $<0,001$ \\
\hline Гипераммониемия, $n$ (\%) & $26(100 \%)$ & 0 & $<0,001$ \\
\hline СРБ, мг/л & $4,1 \pm 2,1$ & $2,6 \pm 1,1$ & 0,003 \\
\hline Гликированный гемоглобин, \% & $6,1 \pm 1,9$ & $5,9 \pm 0,3$ & 0,600 \\
\hline Холестерин, ммоль/л & $6,2 \pm 2,1$ & $6,1 \pm 1,7$ & 0,861 \\
\hline Стеатоз печени S1, $n(\%)$ & 0 & $18(67 \%)$ & $<0,001$ \\
\hline Стеатоз печени S2-S3, $n(\%)$ & $14(54 \%)$ & $6(26 \%)$ & \\
\hline Стеатоз печени S3, $n(\%)$ & $12(46 \%)$ & $2(7 \%)$ & \\
\hline Фиброз печени F1, $n$ (\%) & $26(100 \%)$ & $20(73 \%)$ & 0,030 \\
\hline Фиброз печени F0, $n$ (\%) & 0 & $6(27 \%)$ & \\
\hline $\mathrm{ACT}, \mathrm{ME} / \pi$ & $45,0 \pm 6,7$ & $30,0 \pm 2,2$ & $<0,001$ \\
\hline АЛТ, МЕ/л & $61,0 \pm 3,5$ & $48,0 \pm 2,1$ & $<0,001$ \\
\hline ГГТП, МЕ/л & $89,0 \pm 7,8$ & $49,0 \pm 5,1$ & $<0,001$ \\
\hline Ферритин, мкг/л & $127,0 \pm 3,1$ & $98,0 \pm 1,8$ & $<0,001$ \\
\hline
\end{tabular}

Table 3. Key biomarker dynamics in MAFLD-hyperammonaemic patients at L-ornithine-L-aspartate therapy

\begin{tabular}{|l|c|c|c|}
\hline \multicolumn{1}{|c|}{ Criteria } & Pretherapy & Posttherapy & $p$ \\
\hline Blood ammonia, umol/L & $93 \pm 2.1$ & $53 \pm 4.1$ & $<0.001$ \\
\hline Hyperammonaemia, $n(\%)$ & $26(100 \%)$ & 0 & $<0.001$ \\
\hline CRP, $\mathrm{mg} / \mathrm{L}$ & $4.1 \pm 2.1$ & $2.6 \pm 1.1$ & 0.003 \\
\hline Glycated haemoglobin, \% & $6.1 \pm 1.9$ & $5.9 \pm 0.3$ & 0.600 \\
\hline Cholesterol, mmol/L & $6.2 \pm 2.1$ & $6.1 \pm 1.7$ & 0.861 \\
\hline Liver steatosis S1, $n(\%)$ & 0 & $18(67 \%)$ & $<0.001$ \\
\hline Liver steatosis S2-S3, $n(\%)$ & $14(54 \%)$ & $6(26 \%)$ & \\
\hline Liver steatosis S3, $n(\%)$ & $12(46 \%)$ & $2(7 \%)$ & \\
\hline Liver fibrosis F1, $n(\%)$ & $26(100 \%)$ & $20(73 \%)$ & 0.030 \\
\hline Liver fibrosis F0, $n(\%)$ & 0 & $6(27 \%)$ & \\
\hline AST, IU/L & $45 \pm 6.7$ & $30 \pm 2.2$ & $<0.001$ \\
\hline ALT, IU/L & $61 \pm 3.5$ & $48 \pm 2.1$ & $<0.001$ \\
\hline GGT, IU/L & $89 \pm 7.8$ & $49 \pm 5.1$ & $<0.001$ \\
\hline Ferritin, ug/L & $127 \pm 3.1$ & $98 \pm 1.8$ & $<0.001$ \\
\hline
\end{tabular}

\section{Обсуждение результатов}

Данное исследование

подтвердило, что при МАЖБП у значимой части пациентов имеется гипераммониемия, причем ее наличие было ассоциировано с более высоким значением ИМТ, выраженностью стеатоза и воспаления печени и не зависело от тяжести нарушения обмена липидов и углеводов. Интересно, что у больных с гипераммониемией было выше значение маркера системного воспаления СРБ.
При МАЖБП описано развитие кишечного дисбиоза, избыточного бактериального роста в тонкой кишке и повышение проницаемости кишечного барьера [9]. Все это может приводить к увеличению образования аммиака кишечной микробиотой и усиленному его поступлению в портальной кровоток [9]. Таким образом, мы можем рассматривать гипераммониемию как показатель бактериальной транслокации. Последняя, как известно, приводит к развитию системного воспаления низкой активности, что также наблюдалось в данном 
исследовании [10]. Скорее всего, между повышениями уровней аммиака и СРБ имеется не причинно-следственная связь, а родственная: оба этих процесса являются следствием одного - усиленной бактериальной транслокации.

Аммиак, скорее всего, вносит свой вклад во «второй удар», приводя к повреждению клеток печени, что ведет к ее воспалению, хроническое течение которого имеет закономерным итогом фиброз [1-6].

LOLA не только ожидаемо нормализовал уровень аммиака в крови у таких больных, но и снизил активность маркеров повреждения клеток печени (АЛТ и АСТ), а также уровень стеатоза и фиброза печени и выраженность системного воспаления. Если влияние LOLA на некоторые из данных биомаркеров (АЛТ, АСТ, фиброз) ожидаемо в рамках

\section{Литература / References}

1. European Association for the Study of the Liver (EASL), European Association for the Study of Diabetes (EASD) and European Association for the Study of Obesity (EASO). EASL-EASD-EASO Clinical Practice Guidelines for the management of non-alcoholic fatty liver disease. J Hepatology. 2016;64(6):1388-402. DOI: 10.1016/j. jhep.2015.11.004

2. Ивашкин В.T., Маевская М.В., Павлов Ч.С., Тихонов И.Н., Широкова Е.Н., Буеверов А.О. и др. Клинические рекомендации по диагностике и лечению неалкогольной жировой болезни печени Российского общества по изучению печени и Российской гастроэнтерологической ассоциации. Российский журнал гастроэнтерологии, гепатологии, колопроктологии. 2016;26(2):24-42. [Ivashkin V.T., Mayevskaya M.V., Pavlov Ch.S., Tikhonov I.N., Shirokova Y.N., Buyeverov A.O., et al. Diagnostics and treatment of non-alcoholic fatty liver disease: clinical guidelines of the Russian Scientific Liver Society and the Russian gastroenterological association. Russian Journal of Gastroenterology, Hepatology, Coloproctology. 2016;26(2):24-42 (In Russ.)]. DOI: 10.22416/1382-43762016-26-2-24-42

3. Dasarathy S., Mookerjee RP., Rackayova V., Rangroo Thrane V., Vairappan B., et al. Ammonia toxicity: from head to toe? Metab Brain Dis. 2017;32(2):529-38. DOI: 10.1007/s11011-016-9938-3

4. Qiu J., Tsien C., Thapalaya S., Narayanan A., Weihl C., Ching J., et al. Hyperammonemia-mediated autophagy

\section{Сведения об авторе}

Гаранина Елена Владиславовна - кандидат медицинских наук, гастроэнтеролог, инфекционист ООО «Европейский медицинский центр «УГМК - Здоровье».

Контактная информация: Garanina75@rambler.ru; 620144, г. Екатеринбург, ул. Шейнкмана, д. 113. ORCID: https://orcid.org/0000-0002-2530-9624 нашей теории, то положительный его эффект на другие (СРБ и стеатоз печени) требует дальнейшего ее развития, что составляет основу для последующих исследований.

Ограничением нашего исследования было отсутствие контрольной группы, однако его результаты побуждают провести большое рандомизированное слепое плацебо-контролируемое исследование для верификации полученных результатов.

\section{Заключение}

LOLA в сочетании с модификацией образа жизни, питания и физической активностью нормализует содержание аммиака в крови, уменьшает выраженность воспаления, стеатоза и фиброза печени.

in skeletal muscle contributes to sarcopenia of cirrhosis. A J P Endocrinology and Metabolism. 2012;303(8):98393. DOI: $10.1152 / 00183.2012$

5. Ghallab A., Celliere G., Henkel S.G., Driesch D., Hoehme S., Hofmann U., et al. Model-guided identification of a therapeutic strategy to reduce hyperammonemia in liver diseases. J Hepatology. 2016;64(4):860-71. DOI: 10.1016/j.hepatology.2015.11.018

6. Wilkinson D., Smeeton N.J., Watt P.W. Ammonia metabolism, the brain and fatigue; revisiting the link. Progressin Neurobiology. 2010;91(3):200-19. DOI: 10.1016/j. pneurobio.2010.01.012

7. Gerber L.H., Weinstein A.A., Mehta R., Younossi Z.M. Importance of fatigue and its measurement in chronic liver disease. World J Gastroenterol. 2019;25(28):3669-83. DOI: $10.3748 /$ wjg.v25.i28.3669

8. Ilchenko L.Yu., Nikitin I.G. Hyperammonium in patients with precirrhosis stage: clinical reality? The Russian Archives of Internal Medicine. 2018;8(3):186-93. DOI: 10.20514/2226-6704-2018-8-3-186-193

9. Maslennikov R., Ivashkin V., Efremova I., Poluektova E., Shirokova E. Probiotics in hepatology: An update. World J Hepatol. 2021;13(9):1154-66. DOI: 10.4254 /wjh.v13.i9.1154

10. Maslennikov R., Ivashkin V., Efremova I., Poluektova E., Shirokova E. Gut-liver axis in cirrhosis: Are hemodynamic changes a missing link? World J Clin Cases. 2021;9(31):9320-32. DOI: 10.12998/wjcc.v9.i31.9320

\section{Information about the author}

Elena V. Garanina - Cand. Sci. (Med.), Physician (gastroenterology, infectiology), "UGMK - Zdorovye" European Medical Centre LLC.

Contact information: Garanina75@rambler.ru;

620144, Ekaterinburg, Sheynkmana str., 113.

ORCID: https://orcid.org/0000-0002-2530-9624

Поступила: 13.08.2021 Поступила после доработки: 30.08.2021 Принята: 05.09.2021

Опубликована: 30.09.2021

Submitted: 13.08.2021 Revision received: 30.08.2021 Accepted:05.09.2021 Published: 30.09.2021 